\section{(O) OPEN ACCESS}

\title{
The outcome and cost-effectiveness of nurse-led care in people with rheumatoid arthritis: a multicentre randomised controlled trial
}

\author{
Mwidimi Ndosi, ${ }^{1}$ Martyn Lewis, ${ }^{2}$ Claire Hale, ${ }^{1,3}$ Helen Quinn, ${ }^{3}$ Sarah Ryan, ${ }^{4}$ \\ Paul Emery, ${ }^{5,6}$ Howard Bird, ${ }^{5}$ Jackie Hill ${ }^{1}$
}

Handling editor Tore K Kvien

- Additional material is published online only. To view please visit the journal online (http://dx.doi.org/10.1136/ annrheumdis-2013-203403)

${ }^{1}$ Academic and Clinical Unit for Musculoskeletal Nursing Leeds Institute of Rheumatic and Musculoskeletal Medicine, University of Leeds, Leeds, UK ${ }^{2}$ Arthritis Research UK Primary Care Centre, Keele University, Staffordshire, UK

${ }^{3}$ School of Healthcare,

University of Leeds, Leeds, UK ${ }^{4}$ Haywood Hospital, Stoke-onTrent, UK

${ }^{5}$ Leeds Institute of Rheumatic and Musculoskeletal Medicine, University of Leeds, Leeds, UK ${ }^{6} \mathrm{NIHR}$ Leeds Musculoskeletal Biomedical Research Unit, Leeds Teaching Hospitals NHS Trust, Leeds, UK

\section{Correspondence to} Dr Mwidimi Ndosi, Academic and Clinical Unit for Musculoskeletal Nursing, Leeds Institute of Rheumatic and Musculoskeletal Medicine, 2nd Floor, Chapel Allerton Hospital, Leeds, UK; m.e.ndosi@leeds.ac.uk

Received 5 February 2013 Revised 1 June 2013 Accepted 9 July 2013 Published Online First 28 August 2013
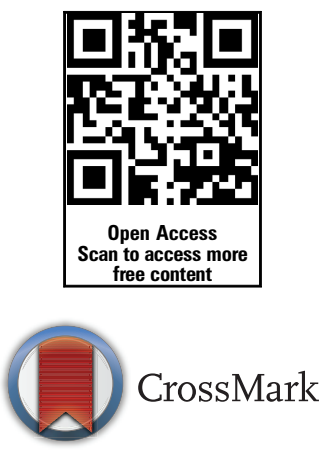

To cite: Ndosi M, Lewis $\mathrm{M}$, Hale C, et al. Ann Rheum Dis 2014;73:1975-1982.

\section{ABSTRACT}

Objective To determine the clinical effectiveness and cost-effectiveness of nurse-led care (NLC) for people with rheumatoid arthritis (RA).

Methods In a multicentre pragmatic randomised controlled trial, the assessment of clinical effects followed a non-inferiority design, while patient satisfaction and cost assessments followed a superiority design. Participants were 181 adults with RA randomly assigned to either NLC or rheumatologist-led care (RLC), both arms carrying out their normal practice. The primary outcome was the disease activity score (DAS28) assessed at baseline, weeks 13, 26, 39 and 52; the non-inferiority margin being DAS28 change of 0.6. Mean differences between the groups were estimated controlling for covariates following per-protocol (PP) and intention-to-treat (ITT) strategies. The economic evaluation (NHS and healthcare perspectives) estimated cost relative to change in DAS28 and quality-adjusted life-years (QALY) derived from EQ5D.

Results Demographics and baseline characteristics of patients under NLC ( $n=91)$ were comparable to those under RLC ( $n=90)$. Overall baseline-adjusted difference in DAS28 mean change $(95 \% \mathrm{Cl})$ for RLC minus NLC was $-0.31(-0.63$ to 0.02$)$ for PP and $-0.15(-0.45$ to $0.14)$ for ITT analyses. Mean difference in healthcare cost (RLC minus NLC) was $£ 710(-£ 352, £ 1773)$ and £128 (-£1263, £1006) for PP and ITT analyses, respectively. NLC was more cost-effective with respect to cost and DAS28, but not in relation to QALY utility scores. In all secondary outcomes, significance was met for non-inferiority of NLC. NLC had higher 'general satisfaction' scores than RLC in week 26 .

Conclusions The results provide robust evidence to support non-inferiority of NLC in the management of RA. Trial registration ISRCTN29803766

\section{INTRODUCTION}

Rheumatoid arthritis (RA) is a chronic inflammatory disease characterised by joint swelling, joint tenderness and destruction of synovial joints, leading to severe disability and premature mortality. ${ }^{1}$ Approximately two million people live with RA in Europe where the average prevalence is $0.49 \% .^{2} \mathrm{RA}$ has a negative impact on individuals' physical, social and psychological functioning, and the resulting disability contributes significantly to the burden of disease in RA. ${ }^{3-6}$ The primary goal of treatment is to suppress disease activity, thus preventing structural damage and optimising function and social participation.

The management of RA has seen significant changes over the past decade due to increased understanding of the disease processes, diagnostic techniques and the development of more efficacious therapies and assessments. The goal of treatment now includes remission, which was once unobtainable at the onset of RA. ${ }^{7}$ This change in the emphasis of treatment has resulted in better health-related outcomes for people with RA, but it has also meant an increased need for monitoring, more coordinated multidisciplinary teams and the development of nurse-led care (NLC). ${ }^{9} 10$

NLC involves adopting a holistic approach taking account of patients' physical, psychological, social and spiritual needs. Nurses involved in providing this service are advanced practitioners in their speciality and function either independently and/or interdependently with other members of the multidisciplinary team. ${ }^{11}$ The role titles clinical nurse specialist (CNS) or rheumatology nurse practitioner are used interchangeably in rheumatology nursing, therefore in this report they are all referred to as CNS. Although the model of NLC was already established in Canada, the USA and Australia in chronic diseases, it was pioneered in the UK in rheumatology services, ${ }^{12}$ and has been shown to be effective, safe and associated with more patient satisfaction. ${ }^{13-16}$ Under this model of care, a patient is referred to NLC following diagnosis and a treatment plan is initiated by the rheumatologist. The role components of NLC include an assessment of disease activity, monitoring effects of therapy, prescribing or recommending medication or dosage changes including intramuscular/ intra-articular steroid injections, provision of patient education, psychological support, providing patients with a contactable knowledgeable and accessible professional through telephone advice lines, coordinating complex care and referring to other health professionals. ${ }^{17}$

In Europe, there are wide variations in the role of the CNS. In The Netherlands and the Scandinavian countries NLC is well established, ${ }^{14-16} 1819$ while in other countries it is in its infancy. ${ }^{20-22}$ The European League Against Rheumatism (EULAR) has recently published recommendations for the role of the nurse in the management of inflammatory arthritis, in order to enable a homogenisation of rheumatology nursing care across Europe. ${ }^{23}$ These 
recommendations support the implementation of NLC and have also identified the need for cost-effectiveness studies of NLC. Although previous UK studies have shown positive results for NLC, they were all single-centre randomised controlled trials (RCT) and focused on clinical effectiveness only. ${ }^{24-27}$ Despite being the pioneer of rheumatology NLC in Europe, the UK has never undertaken an economic evaluation of this model of care until now. The aim of this study was to determine the clinical effectiveness and cost-effectiveness of NLC.

\section{METHODS}

\section{Study design}

This was a multicentre pragmatic RCT. The assessment of clinical effects followed a non-inferiority design, while patient satisfaction and cost-effectiveness assessments followed a superiority design. The study was conducted in outpatient clinics of 10 rheumatology centres across the UK. The study protocol is published elsewhere. ${ }^{28}$

\section{Participants and randomisation}

The inclusion criteria were: a positive diagnosis of RA (1987 American College of Rheumatology (ACR) criteria), ${ }^{29}$ age 18 years or older and the ability to complete questionnaires unaided. Exclusions were: unstabilised concomitant disease, awaiting surgery and already receiving care from the practitioners involved in the study. After gaining patient consent, demographics and disease activity score in 28 joints (DAS28), patients were randomly assigned using a remote secure telephone randomisation service provided by Leeds University Clinical Trials Research Unit. Randomisation was on a $1: 1$ basis to either NLC (experimental group) or rheumatologist-led care (RLC) (control group), by random permuted blocks, using the stratification factors, centre and DAS28 (low disease activity DAS $28 \leq 3.2$, or moderate to high disease activity DAS28 > 3.2). ${ }^{30}$ The independent assessors, performing the joint counts for DAS28, were masked.

\section{Interventions}

This study required three practitioners at each centre; a CNS, a rheumatologist and a blind independent assessor. Nine CNS and 10 rheumatologists delivered the interventions. The CNS had a median experience of 10 years in their current post while the rheumatologists had a median of 9 years at consultant level. The CNS have experience in running nurse-led clinics and usually have a postgraduate qualification in rheumatology nursing and/ or prescribing. Independent assessors were health professionals trained to perform joint counts and calculating the DAS28.

When patients arrived at the clinic, the independent assessor performed 'joint counts' for DAS28 and then oversaw the completion of self-reported pain visual analogue scale (pain-VAS), fatigue-VAS and duration of morning stiffness. The patients were then given questionnaires in 'freepost' return envelopes before consultation with their allocated practitioner. The training of independent assessors was conducted during the study set-up meetings. The rheumatologists and CNS delivering the interventions did not have any special training as they were expected to undertake their 'normal' practice, having agreed to follow the study protocol. Patients were seen by their respective practitioners at baseline and at weeks 13, 26, 39 and 52.

The NLC interventions usually include allocated 30-min time slots in which the CNS takes history, performs physical examination, pain control, prescribing or recommending medication and dosage changes, intra-articular or intramuscular steroid injections, provision of patient education, psychosocial support and ordering blood tests or X-rays. Referrals for ward admission, to the rheumatologist or other health professionals, were carried out as appropriate. The usual RLC is similar to the above except that it usually involves an allocated 15 -min time slot. All interventions, referrals and the duration of the consultation were recorded in a standard 'consultation checklist' designed for this study. Both practitioners saw patients according to the protocol and any extra visits or admissions were recorded.

\section{Outcome measures}

The primary outcome was DAS28 and secondary outcomes were pain severity (pain-VAS), fatigue (fatigue-VAS) and duration of morning stiffness measured at each study visit. Other secondary outcome measures were at baseline, weeks 26 and 52: RA quality of life; ${ }^{31}$ health assessment questionnaire disability index; ${ }^{32}$ hospital anxiety and depression scale; ${ }^{33}$ arthritis self-efficacy scale; ${ }^{34}$ and Leeds satisfaction questionnaire (LSQ). ${ }^{35}$ The health economic questionnaire including EuroQoL (EQ5D) ${ }^{36}$ and healthcare resource data were collected at weeks $0,13,26,39$ and 52. Details of the costs applied to units of resource use are provided in supplementary table S1 (available online only).

\section{Statistical analysis}

Analysis followed per-protocol (PP) and intention-to-treat (ITT) approaches. ${ }^{37}$ The former was based on evaluation of all participants who completed the five treatment sessions and focussed on a complete case analysis; the latter was based on evaluation of all trial participants as allocated. For the ITT approach, missing data were imputed via multiple imputation using chained equations; 20 imputed datasets were computed on guidance that the number needed should approximate the percentage of incomplete cases. ${ }^{38}$

The focus of the evaluation of clinical outcomes was on difference in change scores. A value of 0.6 defined the inferiority/ non-inferiority margin for the primary outcome (DAS28 follow-up change score from baseline), ${ }^{30}$ with the test hypothesis pre-stated as:

- Null hypothesis (inferiority): mean $\triangle \mathrm{DAS} 28_{\mathrm{RLC}}-$ mean $\triangle \mathrm{DAS} 28_{\mathrm{NLC}} \geq 0.6$

where $\Delta=$ change from baseline value.

Allowing for a $10 \%$ participant dropout rate, a total sample size of 180 participants (90 per treatment arm) was needed on the basis of a repeated-measures analysis of between-group differences averaged over four equidistant follow-up time points given $90 \%$ power and one-sided statistical testing with $2.5 \%$ significance level (with anticipated SD of 1.5 , intraclass correlation coefficient of 0.5$).^{30}$

Analysis of the primary endpoint (average $\triangle \mathrm{DAS} 28$ ) and other endpoint comparisons was carried out using linear regression modelling adjusting for age, gender, centre and baseline DAS28 score. Estimates for secondary health outcome measures included additional adjustment for corresponding baseline value. For the $\triangle \mathrm{DAS} 28, \mathrm{p}$ values were generated that tested against the null hypothesis of a between-group difference of 0.6 in favour of RLC. In order to test the secondary outcomes in the same way an equivalent standardised effect size of 0.4 was utilised for the inferiority margin; this level of effect size has been reported to translate to a 'minimal clinically important difference' across typical health-related outcomes. ${ }^{39}$

The null hypothesis of zero difference was used in testing patient satisfaction and cost. The health economic evaluation addressed both cost-effectiveness analysis (in relation to the 
$\triangle \mathrm{DAS} 28$ ) and cost utility (in relation to quality-adjusted lifeyears (QALY) derived from area under the curve calculation of the EQ5D from baseline to last follow-up). Evaluation focused on the incremental cost-effectiveness ratio-ratio of the mean difference in cost to the mean difference in effect (denoting the extra average cost per unit gain in effect); or otherwise dominance of one treatment over the other with respect to lower mean cost and greater mean effect. These estimates were derived through linear regression modelling additionally adjusting for baseline EQ5D. Analysis of uncertainty in the joint estimation of the incremental cost-effectiveness ratio is illustrated via cost-effectiveness planes (via bootstrapping with 5000 replications). Cost-acceptability curves were derived to show the probability of the NLC (experimental treatment) being costeffective at different willingness-to-pay (WTP) thresholds.

Although the primary evaluation was carried out on a priori adjustment for baseline age, gender, centre, DAS28 and EQ5D, a sensitivity analysis was deemed necessary additionally adjusting for baseline biological agent use given the extent of the observed imbalance in that variable at baseline assessment.

\section{RESULTS}

Of the 622 patients who were assessed for eligibility, 181 were eventually randomly assigned and 133 (73.5\%) had complete DAS28 data for all the five visits (PP analysis). The demographics and baseline characteristics of patients under NLC $(n=91)$ were comparable to those under RLC $(n=90)$ except in the proportion of patients receiving biological disease-modifying antirheumatic drugs (DMARD). Figure 1 shows the trial profile and table 1 summarises the baseline characteristics of participants.

Ninety-two per cent (83/90) of patient under NLC attended all five clinic sessions, with a mean total consultation time of 111 min compared to $85 \%$ (77/91) of the RLC group who had a mean total consultation time of $71 \mathrm{~min}$. NLC had longer consultations (median $20 \mathrm{~min}, \mathrm{IQR}=15-30$ vs RLC, 15, 10-15), made fewer medication and dosage changes; ordered fewer intra-articular and intramuscular steroid injections and requested fewer radiological investigations than RLC. There was little difference in the ordering of non-protocol bloods or referrals to other health professionals. NLC provided patient education and psychosocial support more frequently than RLC, and CNS

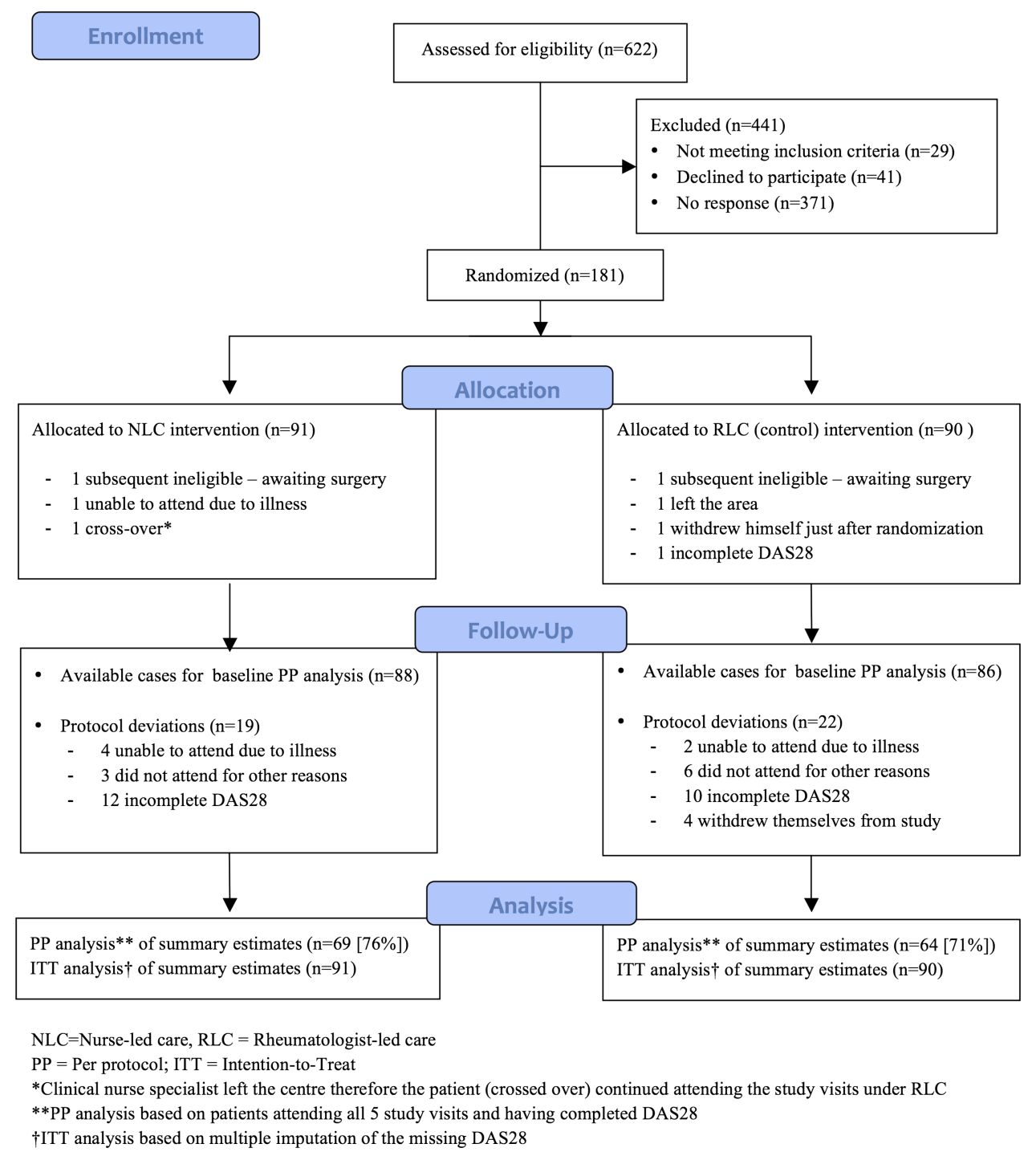

Figure 1 Trial profile. 
Table 1 Baseline characteristics of study population stratified by study group

\begin{tabular}{|c|c|c|}
\hline & $\operatorname{RLC}(n=90)$ & NLC $(n=91)$ \\
\hline Women, $\mathrm{n}(\%)$ & 67 (74.44) & $67(73.63)$ \\
\hline Age, years, mean (SD) & $57.27(12.20)$ & $60.20(11.26)$ \\
\hline Disease duration, years, mean (SD) $(89 ; 90)^{*}$ & $10.21(11.47)$ & $9.57(9.84)$ \\
\hline \multicolumn{3}{|l|}{ Comorbidities, n (\%) } \\
\hline Osteoarthritis & $4(4.44)$ & $5(5.49)$ \\
\hline Osteoporosis & $16(17.78)$ & $9(9.89)$ \\
\hline Hypertension & $19(21.11)$ & $20(21.98)$ \\
\hline Asthma & $7(7.78)$ & $6(6.59)$ \\
\hline Depression & $3(3.33)$ & $1(1.10)$ \\
\hline Hypothyroidism & $6(6.67)$ & $1(1.10)$ \\
\hline \multicolumn{3}{|l|}{ Baseline RA regimen, $\mathrm{n}(\%)$} \\
\hline Methotrexate & $57(63.33)$ & $62(68.13)$ \\
\hline Sulfasalazine & 16 (17.78) & $9(9.89)$ \\
\hline Hydroxychloroquine & $4(4.44)$ & $3(3.30)$ \\
\hline Leflunomide & $6(6.67)$ & $4(4.40)$ \\
\hline Prednisolone & $11(12.22)$ & $6(6.59)$ \\
\hline Biological DMARD & $6(6.67)$ & $15(16.48)$ \\
\hline \multicolumn{3}{|l|}{ Outcomes, mean (SD) } \\
\hline DAS28 $(86 ; 88)^{*}$ & $3.89(1.54)$ & $3.65(1.24)$ \\
\hline Pain $\left(0-100\right.$ VAS, $0=$ no pain) $(86 ; 87)^{*}$ & $39.33(21.26)$ & $43.29(23.77)$ \\
\hline Fatigue $\left(0-100\right.$ VAS, $0=$ no fatigue) $(86 ; 87)^{*}$ & $49.58(25.09)$ & $49.92(24.50)$ \\
\hline Stiffness $\left(0-240\right.$ Min, $0=$ no stiffness) $(84 ; 87)^{*}$ & $50.50(63.42)$ & $43.78(55.50)$ \\
\hline RAQoL $\left(0-30,0=\right.$ better quality of life) $(76 ; 78)^{*}$ & $13.54(7.05)$ & $13.33(8.09)$ \\
\hline $\operatorname{HAQ}(0-3,0=$ no disability $)(86 ; 83)^{*}$ & $1.25(0.76)$ & $1.19(0.77)$ \\
\hline Anxiety $(0-21,0=$ no anxiety $)(87 ; 84)^{*}$ & $7.56(4.24)$ & $7.13(3.83)$ \\
\hline Depression (0-21, $0=$ no depression) $(87 ; 84)^{*}$ & $6.20(3.74)$ & $5.94(3.84)$ \\
\hline ASES $\left(11-110,11=\right.$ poor self-efficacy) $(85 ; 84)^{*}$ & $55.53(17.49)$ & $59.14(18.98)$ \\
\hline \multicolumn{3}{|l|}{ Satisfaction, median (IQR) } \\
\hline LSQ-General (1-5), 1=dissatisfied) $(86 ; 83)^{*}$ & $2.7(2.3,3.4)$ & $2.7(2.3,3.7)$ \\
\hline LSQ-Information (1-5), $1=$ dissatisfied) $(86 ; 83)^{*}$ & $3.2(3.0,3.5)$ & $3.0(2.9,3.7)$ \\
\hline LSQ-Empathy (1-5), $1=$ dissatisfied) $(86 ; 83)^{*}$ & $3.1(3.0,3.6)$ & $3.0(2.9,3.6)$ \\
\hline LSQ-Technical (1-5), 1=dissatisfied) $(86 ; 83)^{*}$ & $3.2(3.0,4.0)$ & $3.1(2.9,4.0)$ \\
\hline LSQ-Attitude (1-5), 1=Dissatisfied) $(86 ; 83)^{*}$ & $3.2(3.0,3.7)$ & $3.2(2.8,3.8)$ \\
\hline LSQ-Access (1-5), $1=$ dissatisfied) $(86 ; 83)^{*}$ & $3.1(3.0,3.6)$ & $3.1(3.0,3.5)$ \\
\hline
\end{tabular}

ASES, arthritis self-efficacy scale; DAS28, disease activity score in 28 joints; DMARD, disease-modifying antirheumatic drug; $\mathrm{HAQ}$ : health assessment questionnaire disability index; LSQ, Leeds satisfaction questionnaire; NLC, nurse-led clinic; RA, rheumatoid arthritis; RAQoL, rheumatoid arthritis quality of life; RLC, theumatologist-led clinic; VAS, visual analogue scale.

*Number of available data were as per randomised allocation that is, 90 for RLC and 91 for NLC unless otherwise stated in parentheses.

conferred more with the rheumatologist than vice versa. NLC had fewer rates of unplanned admissions or visits to the accident and emergency department than RLC. (see supplementary table S2, available online only).

The improvement in disease activity (change in DAS28) over follow-up was better in the NLC group than the RLC group, and significance for non-inferiority was reached in the PP and ITT analyses at all individual follow-up time points and for the primary endpoint (ie, average DAS change). Figure 2 graphically displays the difference between groups in the DAS28 change over the 12-month follow-up period. Table 2 shows the descriptive and inferential results for the DAS28.

Results for the secondary outcomes are shown in supplementary table S3 (available online only). Significance for noninferiority was also reached in all secondary outcomes (at corresponding standardised effect size margins of 0.4 ). Improvements were consistently better for NLC in pain and physical function

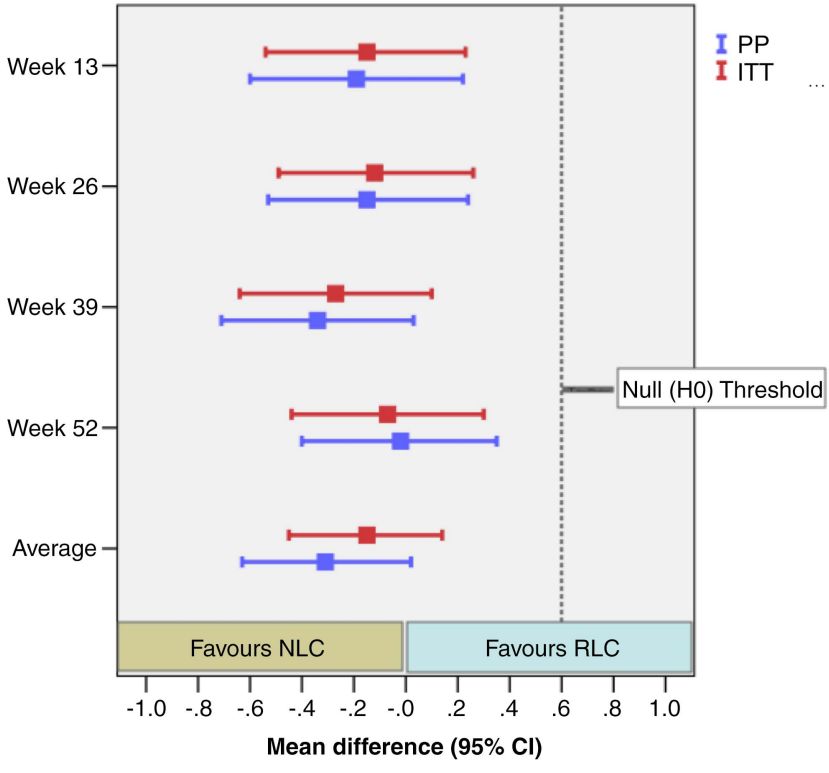

RLC $=$ Rheumatologist-led clinic; NLC $=$ Nurse-led clinic

Mean difference is for RLC group minus NLC group (adjusted for age, gender, Centre and baseline DAS28 score)

Figure 2 Summary estimates for change in disease activity score in 28 joints (DAS28) (primary outcome measure) over 12 months.

outcomes but in fatigue, stiffness and hospital anxiety and depression scale anxiety there was a slight worsening in the NLC compared to slight improvements in the RCL group. In other outcomes, there were some differences between PP and ITT results but all results supported non-inferiority. NLC had higher 'general satisfaction' scores (one subscale of LSQ) than RLC in week 26 , but not in week 52 or other satisfaction subscales, which showed no significant difference between the two groups (see supplementary table $\mathrm{S} 4$, available online only).

NLC has lower consultation costs. While there were no significant differences in the overall mean costs, NLC tended to have lower healthcare costs especially after adjustment for baseline biological agent use (table 3 ). A summary of disaggregated costs is provided in supplementary table S5 (available online only).

Figure 3 shows cost-utility planes and cost-acceptability curves in respect of healthcare costs (similar graphs with respect to NHS costs are provided in supplementary figure S1, available online only). NLC was more cost-effective in respect of costs relative to $\triangle \mathrm{DAS} 28$ for both ITT and PP analyses; therefore, the probability that NLC was cost-effective in relation to the primary outcome was in excess of $80 \%$ across all WTP thresholds. However, in relation to QALY gain, in both healthcare and NHS perspectives, the probability of NLC being cost-effective was dependent of the type of analysis used; favourable in PP but not in ITT analyses (figure 3E and supplementary figure S1E, available online only).

\section{DISCUSSION}

This was the first study to provide information on costeffectiveness of NLC in RA patients with different disease levels. The primary endpoint shows that NLC was not inferior to RLC at any follow-up time point. This suggests that NLC could manage most RA patients without loss of efficacy in terms of disease activity. Disease activity is the best predictor of joint damage and physical disability, both of which can lead to reduction in quality of life and premature mortality. ${ }^{7}$ These results 
Table 2 Summary estimates for change in DAS28 (primary outcome measure) over 12 months

\begin{tabular}{|c|c|c|c|c|c|}
\hline & & $\begin{array}{l}\text { RLC } \\
\text { Mean (SD) }\end{array}$ & $\begin{array}{l}\text { NLC } \\
\text { Mean (SD) }\end{array}$ & $\begin{array}{l}\text { Differencet } \\
\text { Mean }(95 \% \mathrm{Cl})\end{array}$ & p Value* \\
\hline \multirow[t]{2}{*}{ Week 13} & $\operatorname{PP}(73 ; 76)^{*} \ddagger$ & $-0.11(1.48)$ & $-0.04(1.30)$ & $-0.19(-0.60$ to 0.22$)$ & 0.0002 \\
\hline & ITT & $-0.10(1.51)$ & $-0.05(1.36)$ & $-0.15(-0.54$ to 0.23$)$ & $<0.0001$ \\
\hline \multirow[t]{2}{*}{ Week 26} & $\operatorname{PP}(72 ; 80) \ddagger$ & $0.03(1.49)$ & $0.05(1.22)$ & $-0.15(-0.53$ to 0.24$)$ & 0.0001 \\
\hline & ITT & $0.04(1.52)$ & $0.04(1.33)$ & $-0.12(-0.49$ to 0.26$)$ & 0.0001 \\
\hline \multirow[t]{2}{*}{ Week 39} & $\operatorname{PP}(69 ; 76) \ddagger$ & $0.18(1.61)$ & $0.36(1.19)$ & $-0.34(-0.71$ to 0.03$)$ & $<0.0001$ \\
\hline & ITT & $0.20(1.68)$ & $0.33(1.33)$ & $-0.27(-0.64$ to 0.10$)$ & $<0.0001$ \\
\hline \multirow[t]{2}{*}{ Week 52} & $\operatorname{PP}(70 ; 80) \ddagger$ & $0.18(1.41)$ & $0.07(1.22)$ & $-0.02(-0.40$ to 0.35$)$ & 0.0011 \\
\hline & ITT & $0.12(1.50)$ & $0.08(1.32)$ & $-0.07(-0.44$ to 0.30$)$ & 0.0005 \\
\hline \multirow[t]{2}{*}{ Average§ } & $\operatorname{PP}(64 ; 69) \ddagger$ & $0.02(1.32)$ & $0.11(1.05)$ & -0.31 (-0.63to 0.02$)$ & $<0.0001$ \\
\hline & ITT & $0.06(1.32)$ & $0.10(1.10)$ & $-0.15(-0.45$ to 0.14$)$ & $<0.0001$ \\
\hline
\end{tabular}

Table 3 Summary of economic estimates including aggregated cost ( $\mathrm{f}$ ) and effects (QALY and average DAS28 change) estimates

\begin{tabular}{|c|c|c|c|c|c|}
\hline & & $\begin{array}{l}\text { RLC } \\
\text { Mean (SD) }\end{array}$ & $\begin{array}{l}\text { NLC } \\
\text { Mean (SD) }\end{array}$ & $\begin{array}{l}\text { Differencet } \\
\text { Mean }(95 \% \mathrm{Cl})\end{array}$ & p Value* \\
\hline \multicolumn{6}{|l|}{ Costs } \\
\hline \multirow[t]{2}{*}{ Clinic consultations (only) } & PP (69; 79)‡ & $198(57.9)$ & $158(76.7)$ & 34.2 (14.9 to 53.5$)$ & 0.0008 \\
\hline & ITT & $184(79.5)$ & $148(81.2)$ & 35.9 (15.6 to 56.1$)$ & 0.0006 \\
\hline \multicolumn{6}{|l|}{ NHS } \\
\hline \multirow[t]{2}{*}{ (Clinic plus additional NHS resources) } & $\operatorname{PP}(42 ; 50) \ddagger$ & $2171(2822)$ & $1239(2063)$ & $663(-403$ to 1730$)$ & 0.2194 \\
\hline & ITT & $2191(3814)$ & $2282(4277)$ & $-150(-1240$ to 939$)$ & 0.7858 \\
\hline \multicolumn{6}{|l|}{ Healthcare } \\
\hline \multirow[t]{2}{*}{ (NHS resources plus out-of-pocket expenditure) } & PP $(42 ; 50) \ddagger$ & $2286(2793)$ & $1276(2091)$ & $710(-352$ to 1773$)$ & 0.1872 \\
\hline & ITT & $2304(3773)$ & $2386(4565)$ & $-128(-1263$ to 1006$)$ & 0.8245 \\
\hline \multicolumn{6}{|l|}{ Societal } \\
\hline \multirow{2}{*}{ (Healthcare plus productivity loss through time off work) } & $\mathrm{PP}(42 ; 50) \ddagger$ & 2485 (2844) & $1357(2107)$ & $863(-219$ to 1944$)$ & 0.1161 \\
\hline & ITT & 2415 (3785) & $2550(4727)$ & $-195(-1354$ to 963$)$ & 0.7406 \\
\hline \multicolumn{6}{|l|}{ Effects } \\
\hline \multirow[t]{2}{*}{ QALY } & PP (39; 48)‡ & $0.561(0.228)$ & $0.552(0.244)$ & $0.016(-0.049$ to 0.082$)$ & 0.0002 \\
\hline & ITT & $0.575(0.234)$ & $0.554(0.259)$ & $0.020(-0.030$ to 0.071$)$ & $<0.0001$ \\
\hline \multirow[t]{2}{*}{ Average DAS28 change§ } & $\operatorname{PP}(64 ; 69) \ddagger$ & $0.02(1.32)$ & $0.11(1.05)$ & $-0.31(-0.63$ to 0.02$)$ & $<0.0001$ \\
\hline & ITT & $0.06(1.32)$ & $0.10(1.10)$ & $-0.15(-0.45$ to 0.14$)$ & $<0.0001$ \\
\hline \multicolumn{6}{|c|}{ Results of sensitivity test-after additional adjustment for baseline biological agents } \\
\hline \multicolumn{6}{|l|}{ Costs } \\
\hline \multicolumn{6}{|l|}{ NHS } \\
\hline & $\mathrm{PP}(42 ; 50) \ddagger$ & $2171(2822)$ & $1239(2063)$ & $806.05(-111.62$ to 1723.72$)$ & \\
\hline & $\mathrm{ITT}$ & $2191(3814)$ & $2282(4277)$ & 447.67 ( -526.16 to 1421.50$)$ & \\
\hline \multicolumn{6}{|l|}{ Healthcare } \\
\hline & $\operatorname{PP}(42 ; 50) \ddagger$ & $2286(2793)$ & $1276(2091)$ & 852.15 ( -63.37 to 1767.67$)$ & \\
\hline & ITT & $2304(3773)$ & $2386(4565)$ & $494.53(-516.34$ to 15015.41$)$ & \\
\hline \multicolumn{6}{|l|}{ Effects } \\
\hline \multirow[t]{2}{*}{ QALY } & PP (39; 48)‡ & $0.561(0.228)$ & $0.552(0.244)$ & $0.017(-0.049$ to 0.083$)$ & \\
\hline & $\mathrm{ITT}$ & $0.575(0.234)$ & $0.554(0.259)$ & $0.018(-0.034$ to 0.070$)$ & \\
\hline \multirow[t]{2}{*}{ Average DAS28 change§ } & $\mathrm{PP}(64 ; 69) \ddagger$ & $0.02(1.32)$ & $0.11(1.05)$ & $-0.308(-0.640$ to 0.025$)$ & \\
\hline & ITT & $0.06(1.32)$ & $0.10(1.10)$ & $-0.220(-0.5458$ to 0.105$)$ & \\
\hline
\end{tabular}

${ }^{*} p$ Values based on superiority testing (ie, null hypothesis: standardised mean difference $=0.0$ ) in relation to comparison of costs, and non-inferiority testing (ie, null hypothesis: standardised mean difference $=0.4$ ) in relation to comparison of effects.

tDifference in mean DAS28 change scores for the RLC group minus NLC group (adjusted for age, gender, centre, baseline DAS28 score and baseline EQ5D) with CI.

$\ddagger$ Analysis of complete cases (number of responders to resource/time-off work questions-RLC group; NLC group).

$\S$ As presented in table 2 .

DAS28, disease activity score in 28 joints; ITT, intention-to-treat; NLC, nurse-led clinic; PP, per protocol; QALY; quality-adjusted life-year; RLC, rheumatologist-led clinic. 
A. COST-UTILITY PLANE (PP analysis)

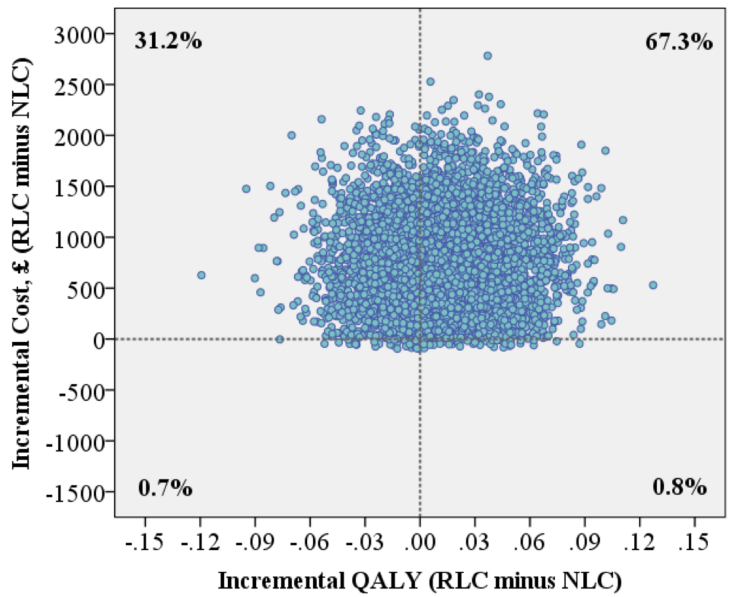

C. COST-EFFECTIVENESS PLANE (PP analysis)

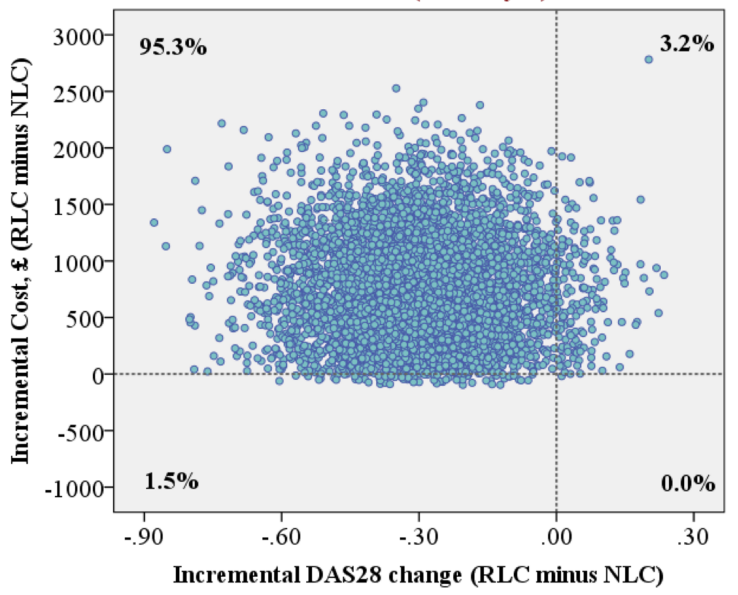

B. COST-UTILITY PLANE (ITT analysis)

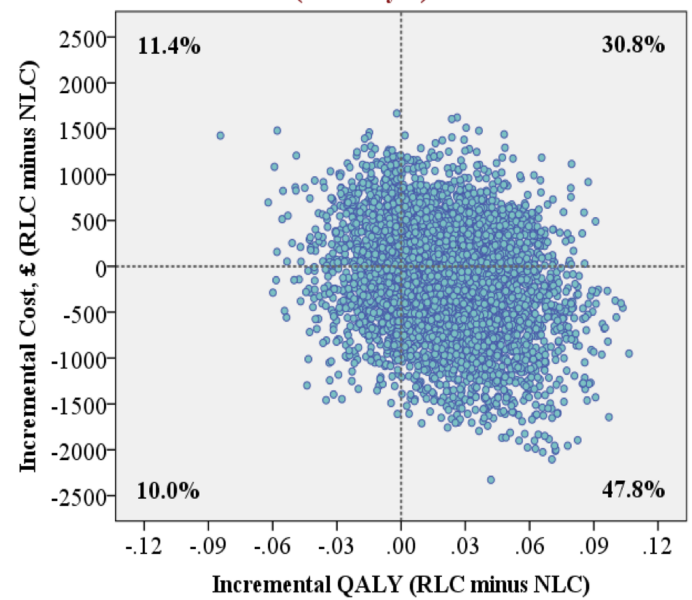

D. COST-EFFECTIVENESS PLANE (ITT analysis)

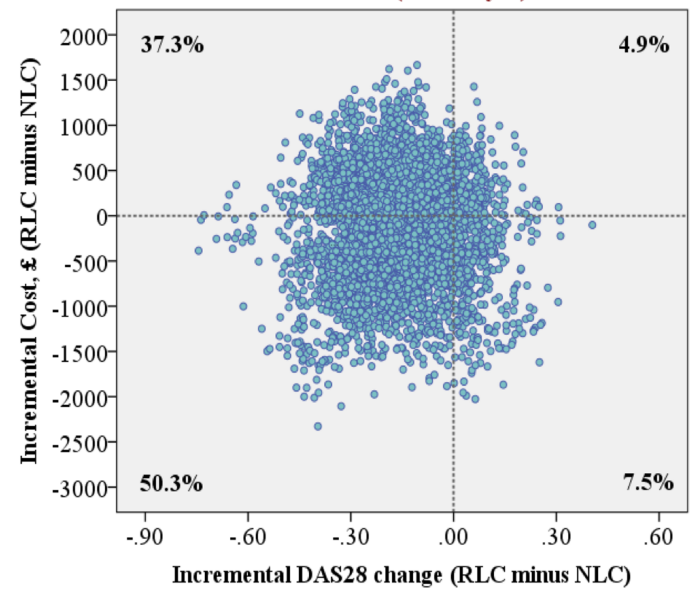

E. COST-EFFECTIVENESS ACCEPTABILITY CURVE

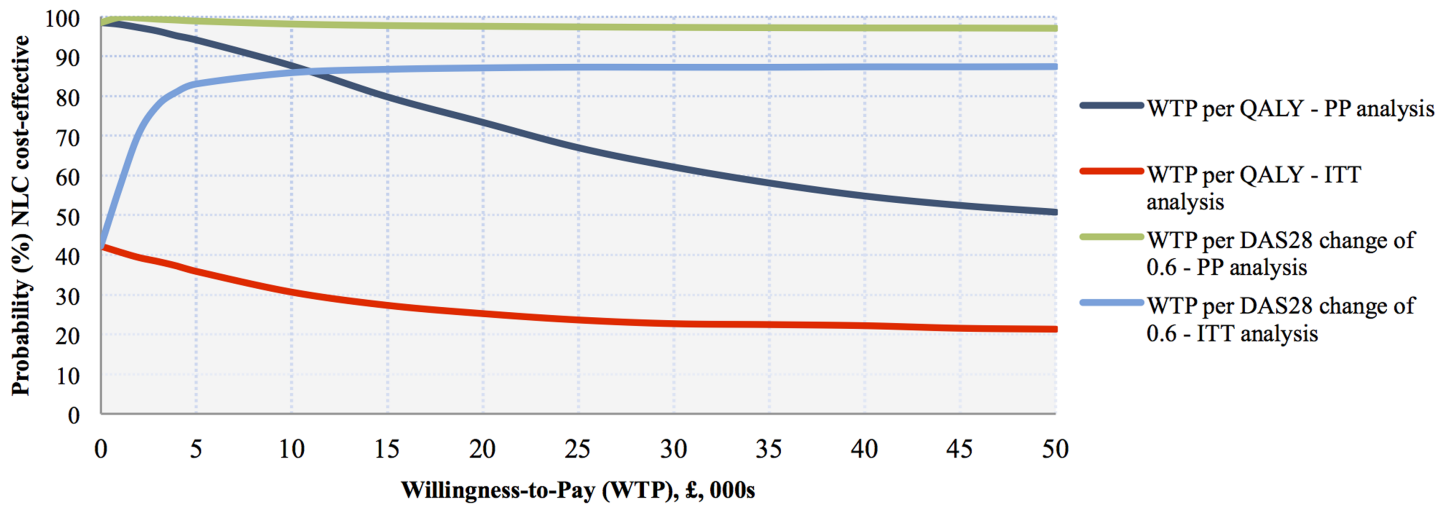

RLC $=$ Rheumatologist-led clinic $;$ NLC $=$ Nurse-led clinic

$\mathrm{PP}=$ Per protocol; ITT $=$ Intention-to-Treat

Incremental health care cost / QALY / DAS28 change values denote bootstrap mean difference for the RLC group minus NLC group (adjusted for age, gender, Centre, baseline DAS28 score and baseline EQ5D)

Percentages in the four quadrants (Graphs A-D) denote the percent of the total (5000) bootstrap cost-effect estimates that fell within each quadrant

Figure 3 Summary of economic evaluation (healthcare perspective).

support this model of care in the UK and are consistent with previous studies in the UK and elsewhere. ${ }^{13-16}$ Whereas other studies have focused on subgroups of patients; for example the Swedish and the Danish studies focused on patients with low disease activity, ${ }^{14-16}$ and the Dutch study on patients with difficulty in performing activities of daily living; ${ }^{18}$ this present study has demonstrated the effectiveness of NLC in managing patients with different disease activity levels.

The baseline difference in the proportion of patients receiving biological DMARD was a result of chance (not systematic). ${ }^{40}$ In the follow-up period, the proportion of patients receiving biological agents in NLC remained more or less constant while that 
in RLC doubled. Assuming that change onto biological agents would significantly improve DAS28, this was likely to favour RLC. Predictably, additional adjustment for baseline biological agents increased the effects on NLC.

The conclusion about the cost-effectiveness of NLC was dependent on the type of outcome; disease specific (DAS28) or generic (QALY) outcome, and on the type of statistical approach used (PP or ITT) in the analysis. The cost-effectiveness of NLC was clearly evident in respect of the primary outcome ( $\triangle$ DAS28) -for example, the estimated probability that NLC is costeffective exceeded $80 \%$ for a cost as little as $£ 5000$ per minimal clinically important difference (ie, per 0.6 change) in DAS28. By contrast, at a guideline WTP threshold of $£ 20$ 000-30 000 per QALY (set by the National Institute for Health and Clinical Excellence), ${ }^{41}$ the probability of NLC being cost-effective was in the range of $62-74 \%$ by PP analysis but $25-27 \%$ by ITT analysis. Given that health policy in the UK is based on generic measures (QALY), ${ }^{42}$ we cannot make strong health policy conclusions about the cost-effectiveness of NLC as the results of QALY (ITT) analysis are inconsistent with the disease-specific measures.

Significance for non-inferiority was reached in all measures although the direction of effects in secondary outcomes did not always favour NLC. For example, NLC giving patient education and psychological support more frequently than RLC did not translate into better improvements in psychological wellbeing outcomes. Understanding the significance of the contribution of each individual outcome in this context is limited because of the interaction effect between outcomes. For example, disease activity is directly related to pain and functional disability; ${ }^{43-45}$ both of which have been shown to determine other outcomes such as quality of life and psychological wellbeing. ${ }^{45}$ Further research on the effect of this interaction is required. Previous RCT have demonstrated more satisfaction with NLC across most LSQ subscales, ${ }^{15} 2527$ but this study has shown this to be the case in 'general satisfaction' only. This study being multicentred might have contributed to this difference. Also, in chronic diseases when some outcomes take a longer time to develop, a person may be 'generally' satisfied with care but still have dissatisfaction with some aspects of it. Patient satisfaction with care is important and may affect adherence to treatment, ${ }^{46}$ therefore there is always room for improvement in a busy clinic.

In this multicentre study, practitioners undertook their 'normal' practice except the frequency of clinic visits, which had to be standardised to ensure comparability between the two groups. Inclusion of patients with different disease levels reflects practice and demonstrates the effectiveness of NLC in managing different groups of patients with RA. In the UK, NLC consultations take place within the rheumatology unit, therefore patients have access to the rheumatologist as required. Based on the number of unplanned admissions, visits to the general practitioner and to the emergency department, there is no reason to question the safety of this model of care.

This study has three main limitations: First, only $73.5 \%$ of the randomly assigned patients had complete data for the primary endpoint in all five visits. The strict criteria for PP analysis was set in order to prevent erroneous claiming of noninferiority. ${ }^{37}$ In the ITT analyses, missing data were inputted using multiple imputation methods, which results in unbiased estimates providing more validity than other approaches to missing data. ${ }^{38} 47$ Second, information on interventions was quantitative (eg, frequencies of conferrals, giving patient education and psychological support) rather than qualitative detail of what was involved. Third, the generalisability of these findings across Europe is limited as NLC effectiveness is likely to be related to the level of its development as a service model.

\section{CONCLUSION}

This study provides robust evidence to support the noninferiority of NLC in managing RA. Indeed, our findings have shown that there may be some clinical benefit of NLC, particularly in respect of disease-specific outcome and general satisfaction with care. In terms of health policy, we are not able to draw firm conclusions on cost-effectiveness given the variation in results between disease-specific and generic outcomes.

Acknowledgements First, the authors would like to express their sincere gratitude to all patients who took part in this trial. Second, they would like to thank Arthritis Research UK who funded the study (grant reference 17632). This study was adopted by UK Clinical Research Network (UKCRN ID 4386) and was provided with infrastructure support in all participating NHS trust hospitals. The authors thank the multicentre study team, the trial support team and the trial steering committee. The multicentre study team comprises Dr Jacqueline Andrews, Mr Zakari Akaribire, Dr Atheer Al-Ansari, Dr Victoria Bejarano, Dr Sarah Bingham, Sr Deborah Bond, Mr Hilary Brownett, Sr Gail Burbage, Sr Deborah Chagadama, Mrs Patricia Cornell, Mrs Elizabeth Gilmore, Dr Sandra Green, Sr Theresa Gripton, Dr Ali Jawad, Miss Lucy Kadiki, Dr Lesley Kay, Sr Clare Kellett, Dr Ken Lim, Mr Hugh Lloyd-Jones, Dr Anne McEntegart, Sr Elizabeth Mclvor, Mrs Steph Mole, Dr Adrian Pace, Dr Fouz Rahmeh, Sr Susan Sisson, Sr Julia Taylor, A Tehseen, Sr Victoria Toner, Sr Jo White, Dr J Williams, Sr Gill Wilson and Mr Tom Wooldridge. The trial support team comprises Ms Helen Greenwood, Mr Glen Saunders and Mrs Jullie Whittle. The trial steering committee (independent members) comprises Mr David Blithe, Mrs Gill Bowskill, Mrs Sarah Fahy and Professor Andy Hassell.

Contributors $\mathrm{JH}$ was the chief investigator and the main grant holder responsible for the original idea and oversaw the project conduct. ML was the study statistician and health economist and was responsible for the protocol development, data analysis, and interpretation of the results and reporting. MN contributed to the protocol development and was responsible for the study set-up, recruiting sites, ethics and research governance applications, study coordinating, interpretation of the results and writing up the manuscripts. $\mathrm{HQ}$, a grant holder, contributed her expertise in planning evaluation of health economics. CH contributed to grant application, supervising the study and reviewing the manuscripts. SR, also a grant holder, contributed to the protocol development, interpretation of the results and reviewing the manuscripts. PE contributed to the grant application, interpretation of the clinical results and reviewing the manuscripts. $\mathrm{HB}$ contributed to the grant application, protocol development, advised on ethics and data monitoring, interpretation of the clinical results and the review of the manuscripts. MN and ML had full access to all the data in the study and take responsibility for the integrity of the data and the accuracy of the data analysis.

Funding Arthritis Research UK funded the study (Grant 17632). The funder was not involved in the preparation of the study protocol, running of the study or preparation of the report. The University of Leeds sponsored the trial, approved the study design and oversaw the overall management of the study.

\section{Competing interests None.}

Ethics approval Multicentre ethics approval was obtained from the Leeds West Research Ethics Committee (MREC ref 06/Q1205/198).

Provenance and peer review Not commissioned; externally peer reviewed.

Open Access This is an Open Access article distributed in accordance with the Creative Commons Attribution Non Commercial (CC BY-NC 3.0) license, which permits others to distribute, remix, adapt, build upon this work non-commercially, and license their derivative works on different terms, provided the original work is properly cited and the use is non-commercial. See: http://creativecommons.org/ licenses/by-nc/3.0/

\section{REFERENCES}

1 Aletaha D, Neogi T, Silman AJ, et al. 2010 Rheumatoid arthritis classification criteria: an American College of Rheumatology/European League Against Rheumatism collaborative initiative. Ann Rheum Dis 2010;69:1580-8.

2 Kobelt G, Kasteng F. Access to innovative treatments in rheumatoid arthritis in Europe. A report prepared for the European Federation of Pharmaceutical Industry Associations (EFPIA). Lunds Universitet; 2009.

3 Bartlett S, Piedmont R, Bilderback A, et al. Spirituality, well being, and quality of life in people with rheumatoid arthritis. Arthritis Care Res 2003;49:778-83.

4 West $E$, Jonsson $S$. Health-related quality of life in rheumatoid arthritis in Northern Sweden: a comparison between patients with early RA, patients with medium-term disease and controls, using SF-36. Clin Rheumatol 2005;24:117-22. 
5 Kobelt G, Lindgren $\mathrm{P}$, Lindroth $\mathrm{Y}$, et al. Modelling the effect of function and disease activity on costs and quality of life in rheumatoid arthritis. Rheumatology (Oxford) 2005;44:1169-75.

6 Pincus T, Callahan LF, Sale WG, et al. Severe functional declines, work disability, and increased mortality in seventy-five rheumatoid arthritis patients studied over nine years. Arthritis Rheum 1984;27:864-72.

7 Smolen JS, Aletaha D, Bijlsma JWJ, et al. Treating rheumatoid arthritis to target: recommendations of an international task force. Ann Rheum Dis 2010;69:631-7.

8 Quinn $\mathrm{M}$, Conaghan P, Emery P. The therapeutic approach of early intervention for rheumatoid arthritis: what is the evidence? Rheumatology (Oxford) 2001;40:1211-20.

9 Deighton C, O'Mahony R, Tosh J, et al. Guidelines: management of rheumatoid arthritis: summary of NICE guidance. BMJ 2009;338:b702.

10 Smolen J, Aletaha D. Monitoring rheumatoid arthritis. Curr Opin Rheumatol 2011;23:252-8.

11 Wong FKY, Chung LCY. Establishing a definition for a nurse-led clinic: structure, process, and outcome. J Adv Nurs 2006;53:358-69.

12 Bird HA. Divided rheumatological care: the advent of the nurse practitioner? Ann Rheum Dis 1983:42:354-5.

13 Ndosi M, Vinall K, Hale C, et al. The effectiveness of nurse-led care in people with rheumatoid arthritis: a systematic review. Int I Nurs Stud 2011;48: 642-54.

14 Primdahl J, Sørensen J, Horn HC, et al. Shared care or nursing consultations as an alternative to rheumatologist follow-up for rheumatoid arthritis outpatients with low disease activity — patient outcomes from a 2-year, randomised controlled trial. Ann Rheum Dis 2014;73:357-64.

15 Koksvik $H S$, Hagen $K B$, Rødevand $E$, et al. Patient satisfaction with nursing consultations in a rheumatology outpatient clinic: a 21 -month randomised controlled trial in patients with inflammatory arthritides. Ann Rheum Dis 2013;72:836-43.

16 Larsson I, Fridlund B, Arvidsson B, et al. Randomized controlled trial of a nurse-led rheumatology clinic for monitoring biological therapy. J Adv Nurs. Published Online First: 17 Jun 2013. doi:10.1111/jan.12183.

17 Leary $A$, Crouch $\mathrm{H}$, Lezard $\mathrm{A}$, et al. Dimensions of clinical nurse specialist work in the UK. Nurs Stand 2008;23:40-4.

18 Tijhuis GJ, Zwinderman AH, Hazes JMW, et al. Two-year follow-up of a randomized controlled trial of a clinical nurse specialist intervention, inpatient, and day patient team care in rheumatoid arthritis. J Adv Nurs 2003:41:34-43.

19 van Eijk-Hustings Y, Ammerlaan J, Voorneveld-Nieuwenhuis $\mathrm{H}$, et al. Patients' needs and expectations with regard to rheumatology nursing care: results of multicentre focus group interviews. Ann Rheum Dis 2013;72:831-5.

20 Jordá $\mathrm{AC}$, Veja MM, Tendero GP, et al. Clinical role for the professional nurse in rheumatology. Reumatología Clín 2008;4:228-31.

21 de la Torre Aboki J. The formation of the Spanish Rheumatology Nursing Society. Musculoskeletal Care 2007:5:1-3.

22 Stamm T, Hill J. Extended roles of non-physician health professionals and innovative models of care within Europe: results from a web-based survey. Musculoskeletal Care 2011;9:93-101.

23 van Eijk-Hustings $Y$, van Tubergen A, Boström C, et al. EULAR recommendations for the role of the nurse in the management of chronic inflammatory arthritis. Ann Rheum Dis 2012;71:13-19.

24 Hill J, Bird HA, Harmer R, et al. An evaluation of the effectiveness, safety and acceptability of a nurse practitioner in a rheumatology outpatient clinic. Br J Rheumatol 1994;33:283-8.

25 Hill J, Thorpe R, Bird H. Outcomes for patients with RA: a rheumatology nurse practitioner clinic compared to standard outpatient care. Musculoskeletal Care 2003;1:5-20.
26 Ryan $S$, Hassell $A B$, Lewis $M$, et al. Impact of a rheumatology expert nurse on the wellbeing of patients attending a drug monitoring clinic. J Adv Nurs 2006:53:277-86

27 Hill J, Lewis M, Bird H. Do OA patients gain additional benefit from care from a clinical nurse specialist?-a randomized clinical trial. Rheumatology (Oxford) 2009;48:658-64

28 Ndosi M, Lewis M, Hale C, et al. A randomised, controlled study of outcome and cost effectiveness for RA patients attending nurse-led rheumatology clinics: study protocol of an ongoing nationwide multi-centre study. Int I Nurs Stud 2011:48:995-1001.

29 Arnett FC, Edworthy SM, Bloch DA, et al. The American Rheumatism Association 1987 revised criteria for the classification of rheumatoid arthritis. Arthritis Rheum 1988;31:315-24.

30 van Gestel A, Haagsma C, van Riel P. Validation of rheumatoid arthritis improvement criteria that include simplified joint counts. Arthritis Rheum 1998:41:1845-50.

31 De Jong Z, Van Der Heijde D, McKenna S. The reliability and construct validity of the RAQoL: a rheumatoid arthritis-specific quality of life instrument. $B r J$ Rheumatol 1997;36:878-83.

32 Kirwan JR, Reeback JS. Stanford health assessment questionnaire modified to assess disability in British patients with rheumatoid arthritis. Rheumatology (Oxford) 1986;25:206-9

33 Zigmon A, Snaith R. The hospital anxiety and depression scale. Acta Psychiatr Scand 1983;67:361-70.

34 Lorig K, Chastain R, Ung E. Development and evaluation of a scale to measure perceived self efficacy in people with arthritis. Arthritis Rheum 1989;32:37-44.

35 Hill J, Bird HA, Hopkins R, et al. Survey of satisfaction with care in a rheumatology outpatient clinic. Ann Rheum Dis 1992;51:195-7.

36 Hurst NP, Kind P, Ruta D, et al. Measuring health-related quality of life in rheumatoid arthritis: validity, responsiveness and reliability of EuroQol (EQ-5D). Rheumatology (Oxford) 1997;36:551-9.

37 Piaggio $\mathrm{G}$, Elbourne $\mathrm{D}$, Altman $\mathrm{D}$, et al. Reporting of noninferiority and equivalence randomized trials: an extension of the CONSORT statement. JAMA 2006;295:1152-60.

38 White IR, Horton NJ, Carpenter J. Strategy for intention to treat analysis in randomised trials with missing outcome data. BMJ 2011;342:910-2.

39 Norman GR, Sloan JA, Wyrwich KW. Interpretation of changes in health-related quality of life: the remarkable universality of half a standard deviation. Med Care 2003;41:582-92

40 Schulz KF, Altman DG, Moher D. CONSORT 2010 statement: updated guidelines for reporting parallel group randomised trials. BMC Med 2010;8:18.

41 NICE. Guide to the methods of technology appraisal. London: National Institute for Health and Clinical Excellence; 2008

42 Eley DS, Del Mar CB, Patterson E, et al. A nurse led model of chronic disease care: an interim report. Aust Fam Physician 2008;37:1030-2.

43 McWilliams DF, Zhang W, Mansell JS, et al. Predictors of change in bodily pain in early rheumatoid arthritis: an inception cohort study. Arthritis Care Res 2012;64:1505-13.

44 Öken Ö, Batur G, Gündüz R, et al. Factors associated with functional disability in patients with rheumatoid arthritis. Rheumatol Int 2008:29:163-6.

45 Welsing PMJ, Van Gestel AM, Swinkels HL, et al. The relationship between disease activity, joint destruction, and functional capacity over the course of rheumatoid arthritis. Arthritis Rheum 2001:44:2009-17.

46 Dang BN, Westbrook RA, Black WC, et al. Examining the link between patient satisfaction and adherence to HIV care: a structural equation model. PloS one 2013;8:e54729.

47 Wright C, Sim J. Intention-to-treat approach to data from randomized controlled trials: a sensitivity analysis. J Clin Epidemiol 2003;56:833-42. 